\title{
DE ALTERIDADES Y MADRIGUERAS DE CONEJO: ESPACIALIDAD Y SIMBOLOGÍA EN LOS CUENTOS FANTÁSTICOS DE JULIO CORTÁZAR
}

\author{
OF ALTERITIES AND RABBIT HOLES: SPACE AND SYMBOLOGY \\ IN THE JULIO CORTÁZAR 'S FANTASY SHORT STORIES
}

\author{
Alberto RODRÍGUEZ BARCÓN \\ Universidade do Porto \\ arbarcon@fe.up.pt
}

Resumen: El artículo propone una revisión crítica de los principales análisis en torno a la dimensión espacial en los cuentos fantásticos de Julio Cortázar hasta la fecha. A partir de este objetivo general, se sugieren dos hipótesis de trabajo. Primero, confirmar aquellas teorías que señalan que el espacio es la estructura vertebral que sostiene su arquitectura fantástica $\mathrm{y}$, segundo, que este espacio interactúa de manera interdependiente y dialógica con otros elementos escénicos hasta configurar una compleja urdimbre de símbolos y códigos que posibilitan el tránsito entre el mundo real y el extraordinario en el imaginario literario cortazariano.

Palabras clave: Cortázar. Espacio. Fantástico. Pasaje.

\begin{abstract}
The article proposes a critical review of the main approaches about the spatial dimension in Julio Cortázar's fantastic stories to date. From this general objective, two working hypotheses are suggested. First, confirm those theories that indicate that space is the vertebral structure that sustains its fantastic architecture and, second, that this space interacts in an interdependent and dialogical way with other scenic elements until it forms a complex warp of symbols and codes that make possible the transit between the real world and the extraordinary one in the cortazariano literary imaginary.
\end{abstract}


Key Words: Cortázar. Space. Fantastic. Passage.

\section{INTRODUCCIÓN}

Son muy numerosos los estudios realizados sobre la obra narrativa de Julio Cortázar, tal y como corresponde a uno de los escritores más reconocidos de la literatura hispanoamericana desde mitad del siglo XX. Estos trabajos abundan, desde diferentes perspectivas, en la poliédrica obra del escritor argentino (Ferro, 2014). La importancia del juego, la transgresión metaliteraria o la complejidad técnica narrativa son algunos de los aspectos formales más estudiados y que evidencian la riqueza del legado literario de Cortázar.

Existe un amplio consenso no sólo en torno a la importancia que la función espacial posee en la narrativa breve del autor, sino en considerar también que el espacio constituye el eje central que sostiene su arquitectura fantástica (Elbanowski, 1992). El diálogo que se articula entre espacio y fantasía posee un fuerte carácter estructurante del desarrollo narrativo. En consecuencia, es imposible hablar del espacio sin analizarlo conjuntamente con la variable fantástica y viceversa, pues la pulsión dialéctica entre ambas dimensiones constituye el andamiaje sobre el que se estructura buena parte de su obra cuentística.

Sin embargo, es reseñable que, si bien la alusión a la espacialidad es una constante en la crítica cortazariana, han sido escasos los intentos de clasificar, de manera estructurada y sistemática, los diferentes roles que el espacio ha jugado en su obra y en la inducción a lo fantástico. Por el contrario, hemos alcanzado, a nuestro parecer, una amalgama de propuestas diversas que, en parte por su reiteración y en parte por aludir a aspectos demasiado sesgados, generan confusión y dificultan articular una visión global del papel que la dimensión espacial juega en sus relatos fantásticos. En este sentido, el presente trabajo propone dos objetivos fundamentales. Por una parte, realizar una revisión de los principales estudios hasta la fecha sobre la espacialidad en la narrativa breve de Cortázar, definiendo y clasificando los principales modelos utilizados por el autor. Por otra, y a partir de este ejercicio inicial, intentar deconstruir y descodificar la compleja interdependencia que el espacio posee en relación con otros 
elementos escénicos y que configura su entramado simbólico.

Rosario Ferré (1990) distingue tres etapas en la trayectoria cuentística de Cortázar. Una primera, calificada como sobrenatural, abarca desde Bestiario hasta Final del juego. La segunda fase, denominada como irracional, engloba a las obras Las armas secretas, Todos los fuegos el fuego y Octaedro. Finalmente, existiría una tercera etapa, de corte social, que se extiende desde Alguien que anda por ahí hasta Deshoras. En el presente trabajo nos ceñiremos, como marco general, a los relatos breves porque es ahí donde la relación entre el espacio y lo fantástico se vuelve más evidente y adquiere múltiples formatos, con especial énfasis en las dos primeras fases señaladas.

\section{EL ESPACIO CORTAZARIANO COMO ESPACIO DE TRANSICIÓN}

Un primer acercamiento a los estudios sobre el espacio en los cuentos de Cortázar permite comprobar que este suele ser presentado frecuentemente como una realidad dual compuesta por la interacción entre dos imaginarios segmentados, pero a la vez indisolubles. Como regla general, el punto de partida del argumento en sus relatos se localiza inscrito en el mundo de lo cotidiano y de la lógica racional. Sin embargo, a medida que la trama avanza, el lector va intuyendo la presencia de otro mundo alternativo y desconocido que obedece a parámetros extraordinarios e irracionales y que termina imponiéndose frente a la descomposición del primero (Goloboff, 2004: 279). El espacio cortazariano aparece así configurado de manera "bidimensional” (Grivel, 1992: 50-57), "contrapuesto" (Amícola, 1997: 4) y "opositivo" (Domínguez, 1991: 57). De este modo, lo fantástico se plasma en un lugar-otro estructuralmente necesario cuyo acceso requiere de superar un determinado desnivel entre espacios (Domínguez, 1991: 58).

Sin embargo, la idea del pasaje posibilita salvar este desnivel entre espacios sin que el lector aprecie el cambio, motivo por el cual Beatriz Sarlo subraya la importancia concreta del pasaje como elemento caracterizador de lo fantástico en Cortázar (1994: 18). De esta manera, el concepto de pasaje, cuyo significado ya había sido recogido con anterioridad cuando Jitrik hablaba de una "zona sagrada" (1971: 52) o Tamborenea de "ruptura de las coordenadas espacio-temporales" (1986: 63), aparece también posteriormente implícito bajo términos como "máquina de pasajes" (Sarlo 
y Saítta, 2007: 265) o "literatura de puentes y pasajes" (Goloboff, 2004: 277), tal y como apunta Suárez (2012: 2733-34).

La habilidad de Cortázar para incorporar de manera fluida y natural lo insólito en lo cotidiano —o lo cotidiano en lo insólito-, se vehicula mediante la resignificación simbólica del espacio. No por casualidad, muchos de sus personajes se encuentran en lugares de transición y de paso como aeropuertos, ascensores, subtes, ómnibus, automóviles o aviones (Rosenblat, 1997: 124). Las connotaciones geográficas sintetizadoras de movimiento han sido también analizadas por Ovares y Rojas en cuentos como "La noche boca arriba", "La isla al mediodía", "Las armas secretas" o "Continuidad de los parques", señalando que las alusiones "hacia el sur, la izquierda, hacia abajo o hacia dentro" connotan lo fantástico, mientras que "lo diurno, la derecha y arriba" representan el mundo real (1999: 13).

A su vez, el concepto de pasaje cortazariano se ha vinculado frecuentemente a la figura geométrica que delinea la cinta de Möbius por su capacidad para conectar el anverso y el reverso de una realidad dual. En este juego narrativo de espacios-espejo, se intuye una suerte de límite inconcreto y permeable que articula la torsión de los planos. Así, frente a la introducción por lo general abrupta y disonante de lo extraordinario en la literatura fantástica tradicional, el desdoblamiento de lo fantástico a partir de una realidad presente en Cortázar se produce sin estridencias (Benedetti, 1967: 88-89; Filinich, 2010; Cepedello y Melendo, 2013). La cotidianeidad y el área imaginada se intersecan mutuamente (Capano, 2014: 109), de manera que el tránsito entre ambas se produce sin que exista una ruptura espacial, expresándose de un modo indeterminado e intersticial (Goloboff, 2004: 279), por lo que no es factible establecer los márgenes que separan la realidad palpable de la imaginaria. A veces el lector lo aprecia por un mero cambio en el enfoque narrativo como en el relato "Axolotl", donde la perspectiva humana pasa a ser una perspectiva animal y el lector comprende así que se ha producido una transmutación de los cuerpos. Por lo tanto, lo fantástico en los cuentos de Cortázar no irrumpe, no se desvela de golpe. Antes bien, se filtra como una distorsión de lo mundano, una inversión sutil de la lógica racional (Ovares y Rojas, 1999: 8; Vidaurre, 2002: 151). El escritor practica una espacialización de lo fantástico como género que opera en el "pasaje de una espacialidad real a otra imaginaria" (Sarlo y Saítta, 2007: 265), imponiendo una lógica que aproxima una sensación de "vacío abismal", en palabras de Lucifora 
(2007: 1).

Sofía Piña, analizando los cuentos de "La puerta condenada", "Continuidad de los parques" y "Una flor amarilla", incluso define esta región transfronteriza que constituye el pasaje a modo de tercer espacio (2013: 1). La propia intercambiabilidad que permite la cinta de Möbius configura una tercera dimensión espacial que no se localiza ni en el anverso ni en el reverso del plano. Un espacio de nadie desenfocado de los parámetros de localización ortodoxos. Piña señala que muchas veces el verdadero horror no aparece cuando ese mundo extraño se muestra, sino cuando existe la posibilidad de encasillarse en el tercer espacio, porque el protagonista sabe que el interregno amorfo que demarca de manera tangencial el mundo concreto del abstracto "no cuadra puramente con ninguna de las dos lógicas de las estructuras temporales presentadas, no puede pensarse a sí mismo desde esa posición alternativa ni construir sentido a partir de ella" (2013: 5). La necesidad de ser encasillado dentro de un sistema, sin importar demasiado que este sea el conocido o el alternativo, obedece a una resignación fatalista por pertenecer a un orden establecido y no permanecer desubicado, y se puede apreciar claramente en relatos como "Una flor amarilla" u "Ómnibus".

Este enfoque fue posteriormente ampliado y complementado por el concepto de lugar desconocido (Pape, 2012: 8). Más allá de actuar como un mero apéndice del pasaje, el lugar desconocido posee relevancia como concepción particular del espacio en obras como "La puerta condenada", en donde si bien el pasaje es importante - simbolizado en la puerta tras el armario- - es el lugar desconocido tras ella, la representación sugestiva del espacio vacío, la que absorbe la atención del lector hasta el final del cuento. Autoras como Béssiere sitúan este vacío sugestivo en la raíz de lo fantástico en los cuentos de Cortázar (1974: 17). El concepto de lugar desconocido se puede asociar con el de territorio insólito de Adam Elbanowski, definido como aquel "trozo del espacio donde sucede un evento que niega las leyes racionales" (1992: 277) y que reconoce en la milonga de "Las puertas del cielo" o las galerías cubiertas de "El otro cielo".

Finalmente, otro enfoque en relación al papel que juega el pasaje en los cuentos cortazarianos es el propuesto por Ovares y Rojas (1999: 10-11), quienes diferencian dos modos de atravesar el umbral. Primero, en función de la "aparición del fantasma", lo otro, lo extraño, que surge del "otro lado" y que remite al hermano muerto en "Cartas de mamá", a 
Celina bailando en "Las puertas del cielo", a lo invasivo en "Casa tomada" o a lo intrusivo en "Continuidad de los parques". Segundo, con base en la "seducción del abismo", donde se impone la presencia de algún elemento que fascina al protagonista y lo atrae al otro plano, como ocurre con la isla paradisíaca en "La isla al mediodía", la obsesiva atracción que siente el protagonista en "Axolotl" o el rol simbólico de la bola de cristal en el pasamanos de la escalera en "Las armas secretas".

\section{UNA REVISIÓN DE LAS PRINCIPALES ESTRUCTURAS ESPACIALES}

Consideramos patente, por lo tanto, que el espacio en los cuentos de Cortázar posee siempre una ambivalencia dialéctica. La pulsión tectónica entre ambas dimensiones produce una tensión narrativa por la que hace aparición lo fantástico. Esta dualidad entre el espacio ordinario y el extraordinario constituye el andamiaje conceptual que articula la espacialidad en sus cuentos y que ha sido catalogada por Eyzaguirre como de "invasión, inversión e interpenetración o fusión de los planos" (1996: 179).

Estableciendo como marco general esta dinámica dual, articulada por el concepto de pasaje como el elemento que vehicula lo extraordinario entre ambos mundos, procedemos a continuación a categorizar otros modos de utilización del espacio más específicos. Lejos de constituir un mero soporte material del argumento, el espacio cortazariano muestra una gran plasticidad interactuando con otros elementos escénicos del relato. Como veremos, esta característica le confiere la capacidad de adoptar diferentes morfologías y diversas valencias simbólicas, construyendo, de este modo, estructuras espaciales que se definen y materializan, por lo general, en contraposición dialéctica a otras estructuras de naturaleza radicalmente opuesta.

\subsection{Espacios cotidianos y espacios imaginarios}

El espacio construido por el imaginario cortazariano es, preferentemente, un espacio urbano. Esta inclinación encaja con la tradición literaria argentina del siglo XX que, representada por autores como Borges, Roberto Arlt o Bioy Casares, prescindió ampliamente del 
mundo rural. La trayectoria vital de Julio Cortázar se encuentra dividida entre las ciudades de Buenos Aires y París y, debido a ello, estos territorios constituyen frecuentemente la localización geográfica de sus relatos (Ainsa, 1973; Schmidt-Cruz, 2000; Herráez, 2014). En algunos casos, como en el cuento "Cartas de mamá", la trama discurre en ambas ciudades, delineando un eje dialógico entre los dos polos. Otras localizaciones también utilizadas por el escritor son Londres ("Instrucciones para John Howell”); París y Roma (“Todos los fuegos el fuego"); valles de Luberon ("Silvia"); Ginebra ("Ahí pero dónde, cómo"); Montevideo ("La puerta condenada"); Cuba ("Reunión") o el Mediterráneo ("La isla al mediodía").

Como no podría ser de otra manera, la ciudad en Cortázar puede ser observada desde diferentes planos simbólicos. Así, por ejemplo, Roger Celis la vincula a una dimensión oculta, prohibida y transgresora del inconsciente que sitúa en un plano onírico (2004: 89). Las referencias topográficas a nombres de calles, líneas de autobús o metro, edificios emblemáticos, etc., son también importantes, pues a menudo enmarcan la acción en localizaciones verosímiles que sitúan al lector en un marco cotidiano formando un efecto de lo real (Barthes, 1968). Un ejemplo evidente lo encontramos en el relato "Ómnibus", donde estas referencias estructuran el contexto urbano que impulsa la trama. Nicolás Rosa (1980: 101) denomina como "itinerarios" a estas estructuras espaciales que median entre zonas y se sobreimprimen sobre caminos ya establecidos. Por otra parte, se hace necesario incorporar también categorías analíticas como el cronotopo, término concebido por Bajtín para analizar como "los elementos de tiempo se revelan en el espacio y [como] el espacio es entendido y medido a través del tiempo" (1989: 238). En este caso, la confluencia del tiempo y del espacio aparece en "Ómnibus" sobreimpuesta por encima de la representación cartográfica de la ciudad de Buenos Aires.

También es pertinente señalar visiones antropológicas dentro del imaginario espacial del viaje desarrolladas por autores como Maradei (2013) o Zygmunt (2017) al analizar las obras de "La autopista del Sur" y Los autonautas de la cosmopista, las dos localizadas en la autopista París-Marsella. En ambas se produce una metamorfosis por la cual este espacio "pensado habitualmente como trayecto, como lugar de tránsito, sufrirá una paulatina transformación hasta adquirir muchos de los rasgos que presenta un espacio vital, donde es posible desarrollar la propia subjetividad y relacionarse con los otros" (Maradei, 2013: 110). Es decir, 
Cortázar propone construir a partir de un no-lugar — concepto definido por el antropólogo francés Marc Augé como un espacio de transición despersonalizado y anónimo generado por la sobremodernidad- un lugar, entendido como un espacio de identidad, relacional e histórico (1994: 83). Naturalmente, existen también cuentos donde no se especifica la localización como en "Con legítimo orgullo", "El viaje", "Continuidad de los parques", "No se culpe a nadie" y "Estación de la mano". En otros casos, el encuadramiento general de la acción es meramente referenciado de manera tangencial sin entrar en más detalles topográficos como en el caso de La Pampa (“Cefalea”).

\subsection{Espacios cerrados y espacios abiertos}

Las localizaciones inscritas en espacios físicamente cerrados poseen un "carácter negativo" (De Mora, 1980: 343) que incrementa la tensión narrativa en el lector y la sensación de asfixia y de restricción del movimiento. Se pueden clasificar en tres tipos: espacios estáticos privados, espacios dinámicos o de transición $\mathrm{y}$, finalmente, espacios estáticos públicos.

En lo que respecta al primer grupo, la casa es un elemento clave en la literatura cortazariana. Por lo general posee una fuerte relevancia como elemento delimitador y categorizador del sujeto en relación con el espacio. Un ejemplo lo podemos observar en "Circe", donde el protagonista es definido hasta una cierta altura del relato siempre como una visita, ajeno hasta entonces a la vida de Delia y a su casa. En "Bestiario", el lector intuye como la casa transpira un ambiente opresivo y coercitivo condicionado por la presencia errante de un tigre que limita la capacidad de movimiento de la familia y una violencia silenciosa y latente en algunas de las relaciones intrafamiliares que sólo la llegada de Isabel consigue quebrar ${ }^{1}$. Otro ejemplo lo observamos en "La salud de los enfermos", donde la acción nucleada en torno a la delicada salud de la madre, y la red de artimañas tejida para preservarla ajena a la accidentada muerte del benjamín de la familia, imprime en el lector la sensación agobiante de un espacio familiar

\footnotetext{
${ }^{1}$ La construcción de una granja de hormigas entre Nino e Isabel, a modo de juego, constituye un espacio paralelo y representativo de la opresión y del peligro de la casa que Rosa denomina como espacio "transicional" (1980: 101).
} 
constreñido.

Sin embargo, si en un relato destaca la presencia de la casa como elemento protagonista y central de la obra es en "Casa tomada" (Sánchez, 2006; De Moraes y Sturzbecher, 2007). El encapsulamiento de los hermanos en la casa les aísla prácticamente del exterior, confinándolos a una rutina austera y absorbente que pivota en torno al cuidado de la misma. Sólo la presencia, apenas sugerida, de un invasor externo consigue expulsarlos al exterior y a un futuro incierto. Más allá de las interpretaciones políticas de este relato (Alazraki, 1994: 72) y de lecturas freudianas del espacio ${ }^{2}$ (Pérez, 1998; Yu-Jin, 2006), la casa constituye un elemento que va más allá del puro formalismo escénico. La casa-protectora del inicio se transforma, sin que el lector entienda exactamente el porqué, en una casa-amenazadora (Valero, 2016: 356). Por lo tanto, el espacio "no es simplemente el marco en el que se desarrolla el relato, es el relato mismo, a partir del cual se configuran todos los elementos narrativos" (Ambriz, 2009: 6).

En el caso de que se trate de un solo personaje, el espacio de la casa se reduce a un departamento ("Carta a una señorita en París"), o apenas una habitación ("No se culpe a nadie", "La puerta condenada" o "El río"). Por otra parte, a veces la casa/habitación no posee ninguna función simbólica más allá de configurar el escenario material de la acción, como sucede en "Las fases de Severo", "El ídolo de las Cícladas", "Estación de la mano" o "Las babas del diablo".

El modelo de espacio cerrado dinámico o de transición aparece reflejado en cuentos como "Manuscrito hallado en un bolsillo", "Una flor amarilla" y "Cuello de gatito negro", donde los transportes públicos concretizan y delimitan la irrupción de lo fantástico. Kipnis considera que el topos del transporte público expresa de una manera concentrada la esencia de la atmósfera del miedo en los relatos de Cortázar (2000: 76). Finalmente, podemos encontrar un tercer grupo localizado en espacios públicos como cines y teatros ("Las Ménades", "Instrucciones para John Howell” y "La banda"), hoteles ("La puerta condenada") u hospitales ("La señorita Cora").

En contraposición al espacio confinado de la casa, los jardines exteriores poseen connotaciones positivas vinculadas al juego y a la adolescencia ("Los venenos", "Bestiario", "Silvia" o "Final del juego").

2 Existen todo tipo de interpretaciones recogidas en Ambriz (2009). 
Sin embargo, el espacio abierto también consigue transmitir en algunos casos una sensación coercitiva como ocurre en "La autopista del Sur", donde un atasco kilométrico en una de las autopistas de entrada a París embotella a cientos de conductores hasta el punto de articular un precario sistema de relaciones sociales entre los viajeros. Pese a la localización exterior, el calor abrasador del verano y el aislamiento espacial provocan una sensación de angustia y de frustración. De manera semejante, la granja de mancuspias en "Cefalea" invoca también un áurea de opresión por la penosa tarea de cuidar a los animales, el aislamiento social y geográfico, y el clima extremo de La Pampa argentina, de manera que la casa asume, en un principio, la función de aliviar la presión abrasiva del exterior.

La interacción entre espacios abiertos y cerrados se produce de manera recurrente en los relatos de Cortázar en un juego de flujos y reflujos bidireccionales orientado a crispar o relajar la trama narrativa. Así, es posible observar movimientos centrípetos en cuentos como "Axolotl" o "Cefalea", donde la dimensión espacial se inicia en una localización semi-abierta y abierta (museo y granja) para acabar trasladándose a una cerrada (pecera y casa/cabeza de los cuidadores), subrayando así la idea de alienación y encarcelamiento.

En otras ocasiones, el sentido de la permeabilidad se vehicula de dentro hacia fuera, como en el caso de "No se culpe a nadie", "Ómnibus" o "La isla al mediodía". Es decir, de una posición estática a otra dinámica. En el primer relato, la opresión que ejerce un simple pulóver - que configura en sí mismo un espacio cerrado- deriva, una vez liberada la cabeza tras una angustiosa gimnasia de giros y contorsiones, en la caída del protagonista por la ventana. En "Ómnibus" la tensión se acumula progresivamente a lo largo del trayecto en bus en tanto que la pareja protagonista subvierte la lógica del espacio (no llevar flores como el resto de los pasajeros). La no participación en el orden preestablecido conjura una suerte de violencia silenciosa, una hostilidad creciente por parte de pasajeros y conductor, de la que solo logran escapar al bajarse en su parada. Finalmente, en "La isla al mediodía" el avión representa la vida presente y cotidiana del protagonista, quien proyecta sobre una isla del Mediterráneo su deseo obsesivo por cambiarla hasta el punto de experimentar una breve estancia en la misma (prototipo de paraíso) por unas horas, momento en que el avión se estrella y el lector asimila que el protagonista realmente nunca salió del mismo. 


\subsection{Alteridad y espacios superpuestos}

La alteridad es una técnica característica de la obra de Cortázar, tanto en lo que se refiere a la duplicidad de personajes como en el caso de la alteridad de espacios en planos y contextos geográficos diversos. Saúl Sosnowski sugiere que al rebelarse los planteamientos espacio-temporales a la lógica ordinaria "se pierden con ello la irreversibilidad del tiempo y la dimensión unívoca del espacio. Se niegan, además, la unidad y la finitud de los hechos y la necesidad teórica de que un hecho sea considerado único en un tiempo y un espacio determinados" (1973: 3).

Carmen de Mora se refiere a esta estructura espacial como "espacios superpuestos" (1980: 348). Desde un punto de vista actancial, el espacio aparece fragmentado en dos escenarios que cohabitan, sino conviven, dentro de la trama argumental del relato, como acontece en el caso de "La noche boca arriba", "Continuidad de los parques", "Siestas", "Recortes de prensa", "El río", "Todos los fuegos el fuego", "Las puertas del cielo", "La isla al mediodía" y "El otro cielo".

La contraposición de espacios dicotómicos a veces se solapa como "realización efectiva de un deseo virtual" (1980: 349) en relatos como "Las puertas del cielo", "La isla al mediodía" y "El otro cielo". En ellos se superponen escenarios alternativos materializados a partir de la proyección obsesiva del protagonista sobre el espacio. En otros casos, la tensión entre planos paralelos sostiene tramas triangulares de amor-odio-celos-rencor como en "Todos los fuegos el fuego", donde el escritor consigue sincretizar dos historias de despecho amoroso emplazadas en contextos geográficos y temporales muy lejanos. Finalmente, en relatos como "La noche boca arriba", el autor propone dos historias ubicadas en territorios y tiempos muy distantes mientras juega al despiste con el lector haciéndole perder la noción entre realidad y sueño.

\subsection{Objetos insólitos, objetos clave y objetos privilegiados}

Como señala Jaime Alazraki, dentro del imaginario fantástico de Cortázar, la realidad alternativa o segunda que se intuye larvada por debajo de la cotidiana precisa, asimismo, de un lenguaje alternativo o segundo que la refiera (1990: 29). Con este propósito, el imaginario literario de 
Cortázar está repleto de objetos metafóricos. Elbanowski apunta que "son elementos que aparecen dentro del marco de lo cotidiano representado y, a la vez, constituyen su componente principal que lleva el espacio a su dimensión fantástica" (1992: 274). Siguiendo la clasificación realizada por Elbanowski, podemos dividir estos elementos fundamentalmente en dos tipos: objetos insólitos y objetos clave.

Dentro del primer grupo, debemos destacar, primeramente, la utilización de algunos animales como herramientas simbólicas para introducir lo fantástico mediante la alteración del orden espacial. Así, por ejemplo, la deambulación — por todos normalizada - de un tigre por la casa en "Bestiario" es una parte integral del espacio; en este sentido, "el tigre no implica ninguna irrupción de lo insólito en lo 'normal', sino que es, justamente, lo cotidiano" (Elbanowski, 1992: 274). Asimismo, los conejos vomitados por el protagonista de "Carta a una señorita en París" también trastornan el orden espacial de un apartamento burgués hasta convertirlo en un territorio caótico. En un nivel inferior, las hormigas también juegan cierto papel en la ordenación espacial del relato en "Los venenos" y "Bestiario". Otro tipo de objeto insólito lo determinan aquellos elementos de la escena que transforman y alteran el espacio. Estos pueden ser materiales, como la estatua de "El ídolo de las Cícladas", que convierte un taller arqueológico en París en un "espacio sagrado", o inmateriales, como los ruidos sordos de "Casa tomada" que, "portadores de un sentido metafórico" (Alazraki, 1990: 29), van acotando y constriñendo el espacio de la casa.

Existe un segundo grupo cuyos elementos son empleados por el autor para desdoblar el espacio, entre los que podemos destacar vidrios, puentes, puertas, ojos, cámaras fotográficas, ventanas, escaleras o galerías (Lizama, 1995: 181). Nicolás Rosa los cataloga como "espacios intersticiales" (1980: 101), donde lo siniestro encuentra la fisura para adentrarse en el mundo cotidiano. Son signos que "nos trasladan al nivel simbólico de la obra" mediante la transmisión de movimiento, por lo que se hallan fuertemente ligados al concepto ya analizado de pasaje, así como de frontera, que estudiaremos más adelante. Estos motivos interactúan con otros elementos narrativos articulando una "red de sentido propio" (Riva, 2007: 62). Elbanowski (1992) destaca, por una parte, el vidrio como categoría espacial que conecta los dos mundos permitiendo la transmutación del personaje como sucede en "La isla al mediodía" o 
en "Axolotl" y, por otra, el puente como punto de enlace entre lo real y lo fantástico en "Lejana", "El otro cielo" y en "Cartas de mamá", donde se alude a un metafórico puente que relaciona el pasado bonaerense y el presente parisino de los protagonistas.

Elbanowski distingue también lo que denomina como objetos privilegiados, situados en un primer plano narrativo, como la bola de vidrio en "Las armas secretas", la estatua en "El ídolo de las Cícladas" o el acuario en "Axolotl”. Se trata de elementos centrales que ensombrecen el resto de la composición espacial del escenario. Un lector atento puede anticipar la sobrevenida de una situación fantástica e intuir que esta pivotará en torno a alguno de estos objetos privilegiados, motivo por el cual estos atrapan, desde un inicio, el foco de atención del lector y subordinan el resto de elementos que configuran la escena.

\subsection{Territorio amenazante y frontera}

Dentro de la heterogeneidad y estratificación del espacio en Cortázar, destaca el concepto de territorio amenazante (Elbanowski, 1992: 277) como una esfera que circunscribe al locus del personaje. Se caracteriza por ser un territorio inhóspito y opresivo que no puede ser atravesado por el protagonista por el peligro y la amenaza que supone. Ejemplos de territorio amenazante son los alrededores de la autopista en "La autopista del Sur", ya que los personajes son inexplicablemente agredidos en cuanto salen de la misma por los habitantes de los pueblos colindantes a la carretera. Las estancias ocupadas por el tigre en "Bestiario" y por lo tanto restringidas al paso, las habitaciones invadidas en "Casa tomada", los anónimos enviados por los vecinos a Mario poniéndole en preaviso en "Circe" o el rechazo de la población y la policía a la granja en "Cefalea" por el miedo a las enfermedades transmitidas por los animales, son muestras de territorios inhóspitos y amenazantes que aíslan el locus del protagonista.

Otro término fundamental para analizar las estructuras espaciales en Cortázar es el concepto de frontera. Elbanowski considera que la frontera "reparte y diversifica el espacio e introduce una serie de contrastes entre territorios separados. En función de la frontera pueden aparecer los requisitos espaciales como el vidrio, pero también una pared o una candileja que separa el escenario de la sala” (1992: 278). Pero, según este autor, la frontera en Cortázar es permeable. Los personajes la atraviesan 
constantemente por dos motivos. Primero, como una actitud deliberada que busca transgredir su locus restringido, suprimiendo el dualismo antagónico entre el mundo real y el mundo evocado. Segundo, por compromiso u obligación en el contexto de un juego o amenaza opresiva. En cualquier caso, la transgresión de la frontera conlleva la transformación del espacio representado y la interpenetración del espacio empírico y el psíquico/ onírico.

Otro investigador que ha estudiado con atención la función de la frontera en los relatos de Cortázar ha sido Mehmet Ilgürel, quien considera que "la frontera es un elemento que trasciende la mera categoría de elemento recurrente y que forma parte de la estructura del cuento" (2017: 62). De este modo, el umbral o frontera es un cronotopo que conecta dos realidades ontológicas diferentes. En su análisis distingue tres modelos de frontera. Primeramente, identifica aquellas fronteras que "vinculan los opuestos" (2017: 58), caracterizadas por confrontar personajes y realidades diferentes, y cuya transgresión suscita lo fantástico. Así, la transgresión de las fronteras dentro-afuera y hombre-animal en "Axolotl"; cariñomaltrato y riqueza-pobreza en "Lejana"; o realidad-delirio en "La noche boca arriba", concluye en un episodio fantástico.

Un segundo modelo de frontera es aquella que "conserva lo extraño" (2017: 59) y que Ilgürel vincula al espacio que configura la casa en los relatos "Casa tomada", "Circe" y "Carta a una señorita en París". En esencia, la casa se transmuta en un personaje central del relato porque delimita y confina la dimensión fantástica del mismo. Por esta razón, cuando los personajes cruzan la frontera y abandonan la casa, lo maravilloso deja de existir. Finalmente, cataloga como frontera-espejo a los umbrales observados en los relatos "La puerta condenada" y "Las armas secretas". En el primero, Ilgürel considera, desde una interpretación psicoanalítica, que los lloros corresponden a la imagen alienada del ánima del protagonista. Asimismo, la ausencia de la bola de vidrio en el segundo relato, motivo recurrente y obsesivo del protagonista en todo el cuento, deriva en un acto de violencia contra su novia, quien representaría nuevamente el ánima del protagonista.

\subsection{EI espacio cognoscitivo y el espacio literario}

Quisiéramos finalizar exponiendo dos enfoques teóricos de 
Carmen de Mora (1980) que, consideramos, presentan un cierto interés a la hora de abordar e interpretar el entramado de códigos y simbologías que Cortázar construye a modo de espacio. Un primer ejemplo es la contextualización del concepto de espacio cognoscitivo de Greimas (1976) en los cuentos fantásticos de Cortázar. Este espacio se define como "un espacio interior que el sujeto se construye para sí mismo, y que por este hecho sólo es significante para él, compuesto de parcelas del saber que ha llegado a adquirir" (Greimas, [1976] 2015: 349). El espacio cognoscitivo se puede reconocer en el mundo imaginativo del protagonista convaleciente en "Liliana llorando", previendo lo que pasará después de su muerte, o en el refugio interior construido por el boxeador "Torito" a partir de la añoranza de éxitos pasados. En "Cartas de mamá" existe también un espacio cognoscitivo prohibido y oscuro entre Luis y Laura en torno a la figura fallecida de Nico, hermano y exnovio, respectivamente, de los protagonistas, que impide romper definitivamente con el pasado (Buenos Aires) y lastra de manera velada el presente (París). Se trata de un espacio intangible y subjetivo construido tácita y silenciosamente sobre el remordimiento de la pareja. Otro ejemplo son las diversas cábalas esgrimidas por Petrone en "La puerta condenada" para explicar los llantos que escucha en su habitación de hotel de madrugada y que también entrarían, según la autora, en esta categoría analítica. Finalmente, en "Ahí pero dónde, cómo" el espacio cognoscitivo se materializa en la calle Rivadavia de Buenos Aires en torno a la enfermedad de Paco, amigo del protagonista muerto treinta años atrás, y que se superpone a través del sueño sobre la realidad ginebrina del narrador.

Una segunda propuesta la constituye la idea de un espacio literario que De Mora identifica en obras como "Bestiario"y "Ahí pero dónde, cómo" y que se concretiza en una literatura que el narrador expone, y mediante la cual el lector puede seguir el curso de sus reflexiones y experiencias, disociada del resto de la narración del relato mediante un uso específico y distintivo de la escritura - utilización de minúsculas, espacios en blanco y frases deshilvanadas - (1980: 353). Incluso, señala De Mora, la propia estructura del relato puede ser representada en términos espaciales. De este modo, relatos como "Axolotl" o "Las babas del diablo" configuran una escritura circular mientras que "Todos los fuegos el fuego" muestran una composición díptica. 


\section{4. ¿HACIA UNA TEORÍA GENERAL DEL ESPACIO EN LOS CUENTOS DE CORTÁZAR?}

Tal y como hemos podido observar hasta el momento, son múltiples los enfoques construidos alrededor del papel que el espacio juega en la narrativa breve de Cortázar. Se trata de una temática sugerente que ha generado una dilatada producción investigadora al respecto. A lo largo de los últimos casi cincuenta años, numerosos investigadores se han sentido atraídos y llamados a explicar cómo funciona esta interacción entre espacio y fantasía. Así, los primeros trabajos pusieron el acento apenas en revelar esta relación y definir, de modo general, sus características más notables. Progresivamente, los análisis sobre esta materia han ido adquiriendo un enfoque más meticuloso y afilado, diseccionando aspectos muy concretos en relación al uso del espacio, y que hemos procedido a estructurar en diferentes categorías a lo largo de este artículo.

Nos hemos centrado, por lo tanto, en recopilar y cotejar, de manera crítica, los diversos enfoques existentes sobre la interacción entre espacio y fantasía en Cortázar. Sin embargo, pese a la extensa literatura especializada al respecto, estas propuestas se centran, de manera general, en apenas media docena de relatos por el peso y la trascendencia narrativa que posee en ellos la dimensión espacial. Estos análisis, sin menoscabo del enorme interés que suscitan, se revelan a la postre como aportaciones deshilvanadas con respecto a una posible visión más amplia y holística del espacio en la extensa narrativa corta de Julio Cortázar. No es, en cualquier caso, momento este para acometer un trabajo tan complejo como la proposición de una teoría estructural del espacio en su obra cuentística. Sin embargo, si es posible subrayar la que, desde nuestro punto de vista, podría ser alguna de sus características fundamentales.

Se ha constatado en este trabajo como la contraposición de dos espacialidades - la real y la irreal — genera una suerte de limbo o frontera que es atravesada por el protagonista permitiendo, así, la aparición de lo fantástico. Sin embargo, consideramos que es posible y pertinente matizar este argumento. De manera general, el espacio mundano, el que se nos presenta con la introducción del relato, es un elemento flexible que se amolda a las andanzas del protagonista (De Mora, 1980). En este sentido, se trata de un espacio subordinado a la acción del personaje, ya que se va construyendo a medida que este interactúa con su entorno inmediato. 
Se caracteriza, por lo tanto, por una naturaleza preferentemente estática y pasiva en relación con el sujeto, quien lo va incorporando a la narración e introduciendo al lector de manera fragmentada y elíptica a medida que la trama avanza.

Frente a este primer modelo espacial se superpone una geografía alternativa cartografiada sobre la lógica transmundana de lo absurdo y lo extraordinario. A diferencia del espacio cotidiano, el imaginario espacial fantástico no es adaptativo. No se amolda a los impulsos y acciones del sujeto protagonista, sino que se impone sobre ellos. Existe a pesar de este y no se define en función de su voluntad, sino que el lector lo percibe como una otra-realidad que subvierte y altera los códigos espaciales tradicionales en los que ambos, personaje y lector, se reconocen y se orientan.

Es aquí donde se presenta, a nuestro parecer, el nudo conceptual de la espacialidad en Cortázar. La confrontación entre estas dos estructuras espaciales propiciaría, según las teorizaciones observadas en este trabajo, la aparición de lo fantástico. Sin embargo, en nuestra opinión, no se trata tanto de una pugna, entendida como un choque entre dimensiones parejas y relativamente equilibradas, como de una imposición incontestable e irrebatible. El imaginario fantástico se superpone fatalmente sobre el mundano, colonizándolo y reconfigurándolo de acuerdo con un nuevo lenguaje geográfico que condiciona, de manera directa, las andanzas y vicisitudes del personaje. En este sentido, podemos hablar de una cadena jerárquica por la cual el espacio ordinario se subordina y reduce al locus del personaje, mientras que este se ve, a su vez, oprimido y restringido por la espacialidad fantástica que, como ya hemos observado, puede adquirir diferentes formas y materializarse en diversas estructuras espaciales.

De este modo, la plasticidad y el carácter pasivo del espacio ordinario quedan supeditados a una nueva conceptualización del mismo que interactúa con el protagonista sin obedecer a su voluntad. Pero el personaje no observa impávido la transmutación de una realidad por otra, sino que intenta asirse, sin conseguirlo, a elementos familiares de referencia. Es en esta maniobra por encuadrar parámetros cognitivos lógicos dentro de una arquitectura espacial incongruente que se adivinan nuevas atribuciones simbólicas y nuevas formas de interactuar con el entorno. Es, en definitiva, donde se desvela la trama fantástica. De este modo, el espacio corriente, vinculado de manera indisoluble al personaje, es asimilado y sometido a la pulsión de un nuevo paradigma espacial que usurpa y trastorna las 
coordenadas geográficas consabidas. La conquista del espacio por lo incoherente y lo extraordinario aboca finalmente al protagonista, en la mayoría de los casos, a envolverse en esa otra espacialidad extraña y traspasar el umbral - a veces atraído, a veces obligado- que revela, finalmente, lo fantástico.

\section{CONCLUSIÓN}

A lo largo de este trabajo ha quedado patente la rica diversidad de interpretaciones y análisis realizados en torno a la dimensión espacial en los cuentos de Cortázar. Son numerosos los intentos por descodificar el complejo juego de signos que cartografían la espacialidad dentro del imaginario fantástico del escritor, y que va más allá de ser el mero soporte material del mismo. El espacio se establece como una pieza central y necesaria dentro del tablero semiótico para intuir —más que entender, pues la ambigüedad es un rasgo característico de su obra - como el orden cotidiano queda subvertido por la infiltración de lo fantástico.

Siendo el tema del espacio un motivo profusamente analizado en los estudios sobre Cortázar, existía, a nuestro entender, bastante confusión sobre los diferentes usos espaciales empleados por el autor en sus relatos fantásticos. Era necesario realizar un punto de situación. En este sentido, el propósito de este trabajo ha consistido en revisitar aquellos análisis literarios en torno a la espacialidad en Cortázar — que Gambetta (1996) desglosa en cuatro dimensiones: la ciudad, la casa, los puentes-pasajes y el viaje - y tratar de reunir, actualizar y clasificar aquellas estructuras espaciales más características dentro de la narrativa breve del escritor.

De esta manera, hemos podido confirmar la tesis, central en muchos análisis, de que el espacio frecuentemente juega un papel transcendental en la aparición de lo fantástico. Para entender esta relación hay que asumir que Cortázar plantea la existencia de una realidad alternativa a la presente, y que dispone la delimitación entre ambas de manera permeable y difusa. El lector, situado inicialmente en un escenario familiar, percibe la expresión de otra lógica que, por entrelíneas de la ordinaria, va imponiéndose de manera hegemónica hasta quedar subsumido en una geografía extraña carente de marcas de orientación.

Hemos clasificado aquellas estructuras espaciales más destacables en diferentes categorías que, no obstante, casi siempre poseen un carácter 
dual que conecta la realidad presente con la alternativa. Todas las estructuras aquí recogidas son, por lo tanto, en esencia, diferentes métodos de interrelacionar el espacio lógico con el ilógico, de puentear lo conocido con lo desconocido, dejando entrever y sobrevenir lo fantástico.

A partir de este primer objetivo de nuestro trabajo, la revisión teórica y la clasificación de las principales estructuras espaciales utilizadas por Cortázar en sus cuentos fantásticos, alcanzamos nuestro segundo propósito: evidenciar la interdependencia que el espacio cortazariano posee en relación con otros elementos de la trama y a partir de la cual se erige un sistema simbólico del que apenas hemos bosquejado algunas claves para su interpretación. Para espacializar lo fantástico, Cortázar se sirve de estos elementos (objetos físicos, animales, elementos sonoros, etc.) a los que confiere un gran capital metafórico y una capacidad para reconfigurar y dotar de nuevos significados al espacio escénico.

Finalmente, a modo de conclusión, se ha puesto el acento en enmarcar la confrontación entre ambas estructuras espaciales, la real y la irreal, en términos de una imposición y colonización de la segunda sobre la primera. De esta manera, redefinimos y desafiamos la tradicional concepción de una confrontación tectónica y relativamente equilibrada entre ambos imaginarios espaciales. Mediante esta dinámica de dominación, se introducen inéditos parámetros de movilidad y se articulan nuevos flujos de interacción dentro del triángulo compuesto por el espacio, los personajes y los elementos escénicos.

Más allá del carácter reflexivo y teórico del presente trabajo, quisiéramos señalar la necesidad de seguir profundizando y continuar proponiendo nuevos enfoques y herramientas analíticas e interpretativas que busquen descodificar el lenguaje velado que podemos apreciar siempre subyacente en sus relatos fantásticos y que alude a un mundo submarino, misterioso y esquivo que transcurre entretejido al nuestro. No obstante, y dado el largo recorrido investigador en torno a la temática espacial al que podemos tener acceso hoy en día, las nuevas propuestas deberían, a nuestro entender, poseer un enfoque estructural e integrarse dentro de una visión general y holística del espacio en Cortázar. 


\section{REFERENCIAS BIBLIOGRÁFICAS}

AINSA, F. (1973). "Las dos orillas de Julio Cortázar". Revista iberoamericana 39.84, 425-456 (también en https://doi. org/10.5195/reviberoamer.1973.2495 [14/11/2018]).

ALAZRAKI, J. (1990). “¿Qué es lo neofantástico?”. Mester 19.2, 21-35. (1994). Hacia Cortázar: aproximaciones a su obra. Barcelona: Anthropos.

AMBRIZ, C. E. (2009). “Análisis narratológico de 'Casa Tomada' de Julio Cortázar”. Espéculo. Revista de estudios literarios 42 (también en:http://webs.ucm.es/info/especulo/numero42/casatoma.html [21/10/2018]).

AMÍCOLA, J. (1997). “'La noche boca arriba' como encrucijada literaria”. Revista iberoamericana LXIII.180, 459-464 (también en https:// doi.org/10.5195/reviberoamer.1997.6206 [22/11/2018]).

AUGÉ, M. (1994). Los “no lugares". Espacios del anonimato. Una antropología de la sobremodernidad. Barcelona: Gedisa Editorial.

BAJTín. M. (1989). Teoría y estética de la novela. Trabajos de investigación. Madrid: Taurus.

BARTHES, R. (1968). "L'effet de réel”. Communications 11, 84-89.

BENEDETTI, M. (1967). Letras del continente mestizo. Montevideo: Arca.

BESSIÈRE, I. (1974). Le récit fantastique. La poétique de l'incertain. Paris: Larousse.

CAMPRA, R. (1996). “Fantasma, ¿estás?”. En Lo lúdico y lo fantástico en la obra de Cortázar: Coloquio Internacional, AA.VV., vol. 1, 213223. Madrid: Fundamentos.

CAPANO, A. D. (2014). "Julio Cortázar y Antonio Tabucchi en dos recreaciones de ambientación griega". Gramma 3, 107-113.

CAPOBIANCO, M. (1994). "Julio Cortázar and the Various Interpretations of Quantum Theory". Taller de letras 22, 7-16 (también en http:// www.bibliotecanacionaldigital.cl/bnd/628/w3-article-205135.html [03/11/2018]).

CELIS, R. (2004). "Julio Cortázar: ciudad, texto y sueño". Confluencia 19, 83-93.

CEPEDELlO MORENO, M. a P. y MELENDO CRUZ, A. (2013). "Narración y sabotaje en 'Las babas del diablo' y 'Blow Up". 
Signa 22, 227-243 (también en http://revistas.uned.es/index.php/ signa/article/view/6351 [14/10/2018]).

DE MORA, C. (1980). "La fijación espacial en los relatos de Cortázar". Cuadernos hispanoamericanos 364-366, 342-353 (también en http://www.cervantesvirtual.com/nd/ark:/59851/ bmcd5166 [09/11/2018]).

DE MORAES, F. e STURZBECHER, T. (2007). "O duplo espacial em 'Casa tomada', de Julio Cortázar". CELLI - Colóquio de Estudos Linguisticos e Literários 3, 380-387.

DOMÍNGUEZ, M. L. (1991). "Del espacio americano al espacio europeo. La espacialidad fantástica en un relato de Adolfo Bioy Casares". Anthropos 127, 57-60.

ELBANOWSKI, A. (1992). "El espacio y lo fantástico en la cuentística de Cortázar". La Palabra y el Hombre 81, 273-278 (también en http:// hdl.handle.net/123456789/1506 [12/11/2018]).

EYZAGUIRRE, L. (1996). "Modos de lo fantástico en cuentos de Julio Cortázar". En Lo lúdico y lo fantástico en la obra de Cortázar: Coloquio Internacional, AA.VV., vol. 1, 177-184. Madrid: Fundamentos.

FERRÉ R. (1990). Cortázar: El romántico en su observatorio. Puerto Rico: Editorial Cultural.

FERRO, R. (2014). "Julio Cortázar entre viajes y bibliotecas". Revista Letral 12, 1-20 (también en http://revistaseug.ugr.es/index.php/ letral/article/view/3767 [12/12/2018]).

FILINICH, M. ${ }^{a}$ I. (2010). "La procedencia incierta de la voz (a propósito de 'Las babas del diablo', de Julio Cortázar". Signa 19, 255272 (también en http://revistas.uned.es/index.php/signa/article/ view/6236/5969[14/10/2018])

GAMBETTA, A. (2011). "Julio Cortázar y el espacio lúdico". Actual Investigación 33, 261-281.

GOLOBOFF, M. (2004). "Una literatura de puentes y pasajes: Julio Cortázar". En Historia crítica de la literatura argentina, S. Saítta (ed.), vol. 9, 277-305. Buenos Aires: Emecé.

GREIMAS, A. J. (1976). Sémantique structurale: Recherche de méthode. Paris: Presses universitaires de France [2015].

GRIVEL, C. (1992). Fantastique-fiction. Paris: Presses Universitaires de France. 
HERRÁEZ, M. (2014). "Referencias parisinas en el imaginario mítico de Julio Cortázar". Revista Letral 12, 21-31 (también en http://revistaseug.ugr.es/index.php/letral/article/view/3768 [14/11/2018]).

ILGÜREL, M. (2017). "Fronteras en el imaginario simbólico de los cuentos de Julio Cortázar". Revista de Literatura, História e Memória 13.22, 53-65 (también en http://e-revista.unioeste.br/ index.php/rlhm/article/view/17454 [02/09/2018]).

JITRIK, N. (1971). El fuego de la especie. Buenos Aires: Siglo XXI.

KIPNIS, L. (2000). "El transporte público como espacio amenazante en tres relatos de Cortázar". Neophilologus 84.1, 75-86.

LIZAMA, P. (1995). "Comentario a la ponencia del profesor Michel F. Capobianco, 'Julio Cortázar and the Various Interpretations of Quantum Theory"'. Taller de letras 23, 177-193.

LUCIFORA, M. C. (2007). "La presencia de lo fantástico en Bestiario, de Julio Cortázar". Espéculo. Revista de estudios literarios 35 (también en http://www.ucm.es/info/especulo/numero35/fanbesti. html [11/12/2018]).

MARADEI, M. G. (2013). "Metamorfosis del espacio urbano y proyecto creador en dos relatos de Julio Cortázar". Question 1.37, 109-123 (también en http://hdl.handle.net/11336/25678 [14/11/2018]).

OVARES, F. y ROJAS, M. (1999). "Espacios de tránsito en los cuentos fantásticos de Julio Cortázar". Letras 31, 5-23.

PAPE, M. (2012). "Puertas y parques y ninguna flor: Un acercamiento al fantástico cortazariano a partir de la espacialidad". Luthor 2.9, $2-10$.

PIÑA, J. S. (2013). "Revisión y alcance del concepto de pasaje como configuración narrativa del espacio en cuentos fantásticos de Julio Cortázar". Silabario. Revista de Estudios y Ensayos Geoculturales 16.XV, 31-48.

PEIRÓ, F. S. (2006). "El espacio en 'Casa tomada' de Julio Cortázar". Literatura: teoría, historia, crítica 8, 195-232 (también en http://www.bdigital.unal.edu.co/14112/1/3-7900-PB.pdf [12/11/2018]).

PÉREZ, V. (1998). “Incesto y espacialización del psiquismo en 'Casa tomada' de Cortázar”. Espéculo: Revista de Estudios Literarios 10 (también en http://www.ucm.es/info/especulo/numero10/cort_poe. 
$h t m l[17 / 11 / 2018])$.

RIVA, S. (2007). "Los motivos recurrentes y la serialización de los relatos en 'Bestiario' de Julio Cortázar'. Analecta Malacitana (AnMal electrónica) 23, 61-71 (también en http://www.anmal.uma.es/ numero23/Bestiario_Cortazar.htm [11/12/2018]).

ROSA, N. (1980). “Julio Cortázar". En Historia de la literatura argentina, AA.VV., vol. 100, 1-24. Buenos Aires: CEAL.

ROSENBLAT, M. L. (1997). Lo fantástico y detectivesco: Aproximaciones comparativas a la obra de Edgar Allan Poe. Caracas: Equinoccio.

SARLO, B. (1994). "Una literatura de pasajes". Espacios de crítica y producción 14, 16-18.

SARLO, B. y SAÍTTA, S. (2007). Escritos sobre literatura argentina. Buenos Aires: Siglo XXI.

SCHMIDT-CRUZ, C. (2000). "De Buenos Aires a París: Los cuentos de Julio Cortázar y la reformulación de su identidad cultural". En Actas del XIII Congreso de la Asociación Internacional de Hispanistas, F. Sevilla Arroyo y C. Alvar Ezquerra (eds.), Vol. III, 411-419. Madrid: Editorial Castalia.

SOSNOWSKI, S. (1973). Julio Cortázar: Una búsqueda mítica. Buenos Aires: Ediciones Noé.

SUÁREZ, N. (2012). "Una literatura de pasajes (y condenas). Notas sobre dos cuentos de Cortázar". En V Congreso Internacional de Letras "Transformaciones Culturales Debates de la teoría, la crítica y la lingüística”, A. Cristófalo y J. Ledesma (eds.), 2733-2739. Buenos Aires: Departamento de Letras, Facultad de Filosofía y Letras (Universidad de Buenos Aires).

TAMBORENEA, M. M. (1986). Julio Cortázar: Todos los fuegos, el fuego. Buenos Aires: Hachette.

TOREA, C. y GUADALUPE, I. (2006). "Julio Cortázar: Cartografías cotidianas". La Colmena 49, 29-39 (también en http://www.redalyc. org/articulo.oa? id=446344560003 [02/12/2018]).

VALERO, E. M. (2016). "Para una poética del espacio (la casa) en el cuento latinoamericano de los 60 a nuestros días: Julio Ramón Ribeyro, Julio Cortázar y Cecilia Eudave". Anales de Literatura Hispanoamericana 45, 343-360 (también en http://revistas.ucm.es/ index.php/ALHI/article/view/551299 [14/12/2018]).

VIDAURRE, C. (2002). "La estructura narrativa en los relatos de Julio 
Cortázar". En Métodos de análisis y análisis de métodos, B. Cárdenas (ed.), 149-165. México: Universidad Michoacana de San Nicolás de Hidalgo.

YU-JIN, S. (2006). "Los espacios de la 'Casa tomada', de Julio Cortázar”. Espéculo: Revista de Estudios Literarios 34, 85-93 (también en http://www.ucm.es/info/especulo/numero34/cortcasa. html [04/12/2018]).

ZYGMUNT, K. (2017). "Llenando de sentido el sinsentido: La transformación del 'no lugar' en lugar antropológico en 'Los autonautas de la cosmopista' o 'Un viaje atemporal París-Marsella' de Julio Cortázar y Carol Dunlop”. En Dimensiones: el espacio y sus significados en la literatura hispánica, R. Crespo y S. Pastor (eds.), 495-506. Madrid: Biblioteca Nueva.

Recibido el 14 de enero de 2019.

Aceptado el 12 de marzo de 2019. 